\title{
Holographic Duals of Argyres-Douglas Theories
}

\author{
Ibrahima Bah, ${ }^{1}$ Federico Bonetti $\odot,{ }^{2}$ Ruben Minasian $\odot,{ }^{3}$ and Emily Nardoni $\odot{ }^{4}$ \\ ${ }^{1}$ Department of Physics and Astronomy, Johns Hopkins University, 3400 North Charles Street, Baltimore, Maryland 21218, USA \\ ${ }^{2}$ Mathematical Institute, University of Oxford, Woodstock Road, Oxford OX2 6GG, United Kingdom \\ ${ }^{3}$ Institut de Physique Théorique, Université Paris Saclay, CNRS, CEA, F-91191, Gif-sur-Yvette, France \\ ${ }^{4}$ Mani L. Bhaumik Institute for Theoretical Physics, Department of Physics and Astronomy, University of California,
}

Los Angeles, California 90095, USA

(Received 10 August 2021; accepted 26 October 2021; published 15 November 2021)

\begin{abstract}
We propose the first explicit holographic duals for a class of superconformal field theories of ArgyresDouglas type, which are inherently strongly coupled and provide a window onto remarkable nonperturbative phenomena (such as mutually nonlocal massless dyons and relevant operators of fractional dimension). The theories under examination are realized by a stack of $M 5$-branes wrapped on a sphere with one irregular puncture and one regular puncture. In the dual $11 d$ supergravity solutions, the irregular puncture is realized as an internal M5-brane source.
\end{abstract}

DOI: 10.1103/PhysRevLett.127.211601

Introduction.-Strong-coupling phenomena in quantum field theory (QFT) are of crucial importance, both conceptually and phenomenologically, but their study poses considerable theoretical challenges. In the endeavor of exploring the vast and largely uncharted landscape of strongly coupled phases in QFT, valuable lessons can be learned from theories with a higher degree of symmetry. Superconformal field theories (SCFTs) of Argyres-Douglas (AD) type in four dimensions constitute a prominent example. These theories are intrinsically strongly coupled and describe interactions among mutually nonlocal massless dyons [1]. Their spectrum contains relevant Coulomb branch operators of fractional dimension. Establishing the existence and surprising properties of these QFTs has been complicated by their lack of an $\mathcal{N}=2$ weak-coupling Lagrangian description, and hence, exploring less conventional windows into their physics is especially valuable.

A vast class of SCFTs of AD type is expected to admit holographic duals, but their identification has remained an open problem for years. In this Letter, we present a new class of fully explicit $\mathrm{AdS}_{5}$ solutions in $11 d$ supergravity and we propose them as holographic duals to SCFTs of AD type. Our results give the opportunity to analyze these QFTs from a new angle, providing novel insights on their properties. Furthermore, a subclass of SCFTs of AD type can be realized as $\mathcal{N}=2$ supersymmetric IR fixed points of renormalization group $(\mathrm{RG})$ flows preserving $\mathcal{N}=1$

Published by the American Physical Society under the terms of the Creative Commons Attribution 4.0 International license. Further distribution of this work must maintain attribution to the author(s) and the published article's title, journal citation, and DOI. Funded by SCOAP. supersymmetry $[2,3]$. Our solutions pave the way to the exciting possibility of studying the gravity dual of supersymmetry enhancing RG flows, which could shed new light on holography in general.

A crucial feature of our $\mathrm{AdS}_{5}$ solutions is the presence of suitable singularities, which we interpret as the low-energy approximation to well-defined brane sources in $M$ theory. Localized sources in the internal space constitute an important ingredient in the holographic dictionary that allows for arbitrary flavor symmetries (see, e.g., [4-11]). This Letter describes novel controlled examples allowing a better understanding of these sources, pivotal for enlarging the scope of the AdS/CFT correspondence.

Supergravity solutions.-Our $\mathrm{AdS}_{5}$ solutions in $11 d$ supergravity preserve $4 d \mathcal{N}=2$ superconformal symmetry. They were obtained in $7 d$ gauged supergravity and uplifted on $S^{4}$, as will be reported in [12]. The $7 d$ solutions are a warped product of $\mathrm{AdS}_{5}$ and a $2 d$ space $\Sigma$, consisting of a circle fibered over an interval. $\Sigma$ is supported by a $\mathrm{U}(1)$ gauge flux, does not have a constant curvature metric, and admits a nonconstant Killing spinor. Thus, as in [13-15], supersymmetry is not realized in the standard topological twist paradigm.

The metric of the uplifted $11 d$ solution is

$$
\begin{gathered}
d s_{11}^{2}=m^{-2} e^{2 \lambda}\left(d s_{\mathrm{AdS}_{5}}^{2}+d s_{M_{6}}^{2}\right), \\
d s_{M_{6}}^{2}=\frac{d w^{2}}{2 w h(w)\left(1-w^{2}\right)^{3 / 2}}+\frac{\mathcal{C}^{2} h(w) d z^{2}}{B} \\
+\frac{\sqrt{1-w^{2}}}{2 B}\left[\frac{d \mu^{2}}{w\left(1-\mu^{2}\right)}+\frac{\left(1-\mu^{2}\right) D \phi^{2}}{w \mathcal{H}(w, \mu)}+\frac{w \mu^{2} d s_{S^{2}}^{2}}{\mathcal{H}(w, \mu)}\right],
\end{gathered}
$$


where $m$ is a mass scale, $d s_{\text {Ads }}^{2}$ is the metric on the unitradius $\mathrm{AdS}_{5}$, and $d s_{S^{2}}^{2}$ is the metric on the unit-radius $S^{2}$. The functions $h(w), \mathcal{H}(w, \mu)$ are defined as

$$
h=B-2 w \sqrt{1-w^{2}}, \quad \mathcal{H}=\mu^{2}+w^{2}\left(1-\mu^{2}\right),
$$

where $0<B<1$ is a constant parameter. The coordinates $\mu, w$ have ranges $0 \leq \mu \leq 1$ and $0 \leq w \leq w_{1}$, with $w_{1}^{2}=\frac{1}{2}\left(1-\sqrt{1-B^{2}}\right)$. The angular coordinates $\phi, z$ have period $2 \pi$, and $\mathcal{C}$ is a constant. The 1 -form $D \phi$ and the warp factor are given by

$D \phi=d \phi+\mathcal{C}\left(2 w^{2}-1\right) d z, \quad e^{2 \lambda}=\frac{2 B w^{1 / 3} \mathcal{H}^{1 / 3}}{\sqrt{1-w^{2}}}$.

The $G_{4}$ flux supporting the solution reads

$$
G_{4}=-\frac{1}{m^{3}} \operatorname{vol}_{S^{2}} \wedge d\left[\frac{\mu^{3} D \phi}{\mathcal{H}}\right],
$$

where $\operatorname{vol}_{S^{2}}$ is the volume form of the $S^{2}$.

The space $M_{6}$ is an $S_{z}^{1} \times S_{\phi}^{1} \times S^{2}$ fibration over the rectangle $\left[0, w_{1}\right] \times[0,1]$ in the $(w, \mu)$ plane, see Fig. 1 . The directions $w, S_{z}^{1}$ in (2) are identified with $\Sigma$ in the $7 d$ solution, while $\mu, S_{\phi}^{1}, S^{2}$ span the $S^{4}$ used in the uplift.

Regularity and flux quantization.-As we approach a point in the interior of the $\mathrm{P}_{1} \mathrm{P}_{2}$ segment in the $(w, \mu)$ plane (see Fig. 1), the $S^{2}$ shrinks smoothly. The Killing vector $\partial_{\phi}$ shrinks smoothly in the interior of $\mathrm{P}_{3} \mathrm{P}_{4}$. The linear combination $\partial_{\phi}+\ell \partial_{z}$ shrinks smoothly along $\mathrm{P}_{2} \mathrm{P}_{3}$, where $\ell$ is given as

$$
\ell=\frac{1}{\mathcal{C} \sqrt{1-B^{2}}}, \quad \ell \in \mathbb{N} .
$$

The quantization of $\ell$ stems from analyzing the local geometry of the $4 d$ space spanned by $w, \mu, \phi, z$ near $\mathrm{P}_{3}$, and requiring it to be locally an orbifold $\mathbb{R}^{4} / \mathbb{Z}_{\ell}$.

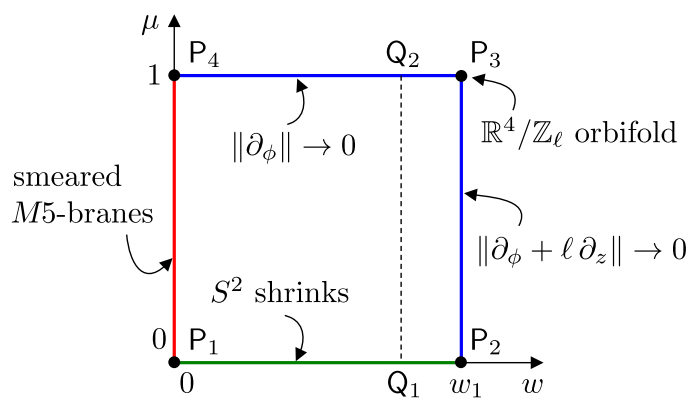

FIG. 1. The internal space is an $S_{\phi}^{1} \times S_{z}^{1} \times S^{2}$ fibration over $\left[0, w_{1}\right] \times[0,1]$ in the $(w, \mu)$ plane. The $S^{2}$ shrinks smoothly along $\mathrm{P}_{1} \mathrm{P}_{2}$. Different linear combinations of $\partial_{\phi}, \partial_{z}$ shrink smoothly along $\mathrm{P}_{2} \mathrm{P}_{3}$ and $\mathrm{P}_{3} \mathrm{P}_{4}$, as indicated. At $\mathrm{P}_{3}$ the $4 d$ space parametrized by $w, \mu, \phi, z$ is locally $\mathbb{R}^{4} / \mathbb{Z}_{\ell}$. The region near $\mathrm{P}_{1} \mathrm{P}_{4}$ is interpreted in terms of smeared $M 5$-branes. The segment $Q_{1} Q_{2}$ enters the definition of the four-cycle $\mathcal{C}_{4}$.
The internal space $M_{6}$ admits nontrivial four cycles which lead to flux quantization conditions for $G_{4}$. The fourcycle $\mathcal{C}_{4}$ is obtained by combining the segment $\mathrm{Q}_{1} \mathrm{Q}_{2}, S_{\phi}^{1}$, and $S^{2} \cdot \mathcal{C}_{4}$ has the topology of a four sphere because the $S^{2}$ shrinks at $Q_{1}$ and the $S_{\phi}^{1}$ shrinks at $Q_{2}$. The flux of $G_{4}$ through $\mathcal{C}_{4}$ with suitable orientation defines

$$
N=\int_{\mathcal{C}_{4}} \frac{G_{4}}{\left(2 \pi \ell_{p}\right)^{3}}=\frac{1}{\pi m^{3} \ell_{p}^{3}}, \quad N \in \mathbb{N},
$$

where $\ell_{p}$ is the $11 d$ Planck length. Next, we define the four-cycle $\mathcal{B}_{4}$ by combining $S^{2}$, the segment $\mathrm{P}_{2} \mathrm{P}_{3}$, and the linear combination of $S_{\phi}^{1}$ and $S_{z}^{1}$ that does not shrink in the interior of $\mathrm{P}_{2} \mathrm{P}_{3} \cdot \mathcal{B}_{4}$ is topologically a four sphere, because the $S^{2}$ shrinks at $\mathrm{P}_{2}$ and both $S_{\phi}^{1}$ and $S_{z}^{1}$ shrink at the orbifold point $\mathrm{P}_{3}$. For $\ell=1$ we have $\mathcal{B}_{4} \cong \mathcal{C}_{4}$, but $\mathcal{B}_{4}$ is an independent four cycle for $\ell>1$, with

$$
\mathbb{Z} \ni \int_{\mathcal{B}_{4}} \frac{G_{4}}{\left(2 \pi \ell_{p}\right)^{3}}=\frac{N}{\ell}, \quad \text { hence } \ell \text { divides } N .
$$

Finally, we construct the four-cycle $\mathcal{D}_{4}$ by combining $\mathrm{P}_{3} \mathrm{P}_{4}$ with $S^{2}$-which shrinks at $\mathrm{P}_{4}$ —and the combination of $S_{\phi}^{1}$ and $S_{z}^{1}$ that does not shrink in the interior of $\mathrm{P}_{3} \mathrm{P}_{4}$. Integrating $G_{4}$ on $\mathcal{D}_{4}$ defines

$K=\int_{\mathcal{D}_{4}} \frac{G_{4}}{\left(2 \pi \ell_{p}\right)^{3}}=\frac{N\left(1-\sqrt{1-B^{2}}\right)}{\ell \sqrt{1-B^{2}}}, \quad K \in \mathbb{N}$.

In the vicinity of $\mathrm{P}_{1} \mathrm{P}_{4}$, the geometry is singular and $e^{2 \lambda}$ vanishes. We interpret this in terms of a smeared M5-brane source, as inferred from $G_{4}$ near $w=0$,

$$
G_{4}=-\frac{\operatorname{vol}_{S^{2}} \wedge d \mu \wedge D \phi}{m^{3}}+\cdots .
$$

This term yields a finite flux equal to $N$ (7) when integrated along $S^{2}, \mu, S_{\phi}^{1}$, signaling a source of the form $d G_{4} \sim \delta(w) d w \wedge \operatorname{vol}_{S^{2}} \wedge d \mu \wedge D \phi$. Comparing the $11 d$ metric near $w=0$ with the standard M5-brane solution, we see that the $M 5$-branes are extended along $\mathrm{AdS}_{5}$ and the $S_{z}^{1}$, smeared along the $\mu, S_{\phi}^{1}$ directions, and sitting at the origin $w=0$ of the local $\mathbb{R}^{3}$ space $d w^{2}+w^{2} d s_{S^{2}}^{2}$.

Solutions in canonical $\mathcal{N}=2$ form. - The general form of an $\mathrm{AdS}_{5} \quad M$-theory solution preserving $4 d \mathcal{N}=2$ superconformal symmetry was determined in [16] by Lin, Lunin, and Maldacena (LLM). The $11 d$ metric and flux are summarized in [17]. In LLM form, the internal space $M_{6}$ is an $S_{\chi}^{1} \times S^{2}$ fibration over a $3 d$ space with local coordinates $\left(x_{1}, x_{2}, y\right)$. The Killing vector $\partial_{\chi}$ is associated with the $\mathrm{U}(1)_{r} R$ symmetry of the dual SCFT, while the isometries of the $S^{2}$ are mapped to the $\mathrm{SU}(2)_{R} R$ symmetry. The solution is determined by a function $D\left(x_{1}, x_{2}, y\right)$ satisfying the Toda equation 


$$
\left(\partial_{x_{1}}^{2}+\partial_{x_{2}}^{2}\right) D+\partial_{y}^{2} e^{D}=0 .
$$

Our solutions can be cast in canonical LLM form, with the $S^{2}$ in (2) identified with the $S^{2}$ in LLM. Defining $x_{1}+i x_{2}=r e^{i \beta}$, the map between $\chi, \beta$ and $\phi, z$ is

$\partial_{\chi}=\partial_{\phi}+\frac{N \ell}{N+K \ell} \partial_{z}, \quad \partial_{\beta}=\partial_{\phi}+\left[1+\frac{N \ell}{N+K \ell}\right] \partial_{z}$.

With reference to the uplift from $7 d$, the isometry $\partial_{\chi}$ mixes the $\Sigma$ and $S^{4}$ directions. This is in contrast to the solutions of $[17,18]$, in which $\partial_{\chi}=\partial_{\phi}$.

The function $D$ and the map between the LLM coordinates $y, r$ and the coordinates $\mu, w$, are

$$
\begin{aligned}
y & =\frac{4 B w \mu}{\sqrt{1-w^{2}}}, \quad r=\left(1-\mu^{2}\right)^{-1 / 2 \mathcal{C}} \mathcal{G}(w), \\
e^{D} & =\frac{16 B \mathcal{C}^{2}\left(1-\mu^{2}\right)^{1+(1 / \mathcal{C})} h}{\left(1-w^{2}\right) \mathcal{G}(w)^{2}}, \frac{\mathcal{G}^{\prime}(w)}{\mathcal{G}(w)}=\frac{-B w}{\mathcal{C}\left(1-w^{2}\right) h} .
\end{aligned}
$$

This determines a class of exact solutions $D$ to the Toda equation (11) which are separable in the variables $\mu, w$. Crucially, in our setup $D$ does not describe a constant curvature Riemann surface, in contrast to the $4 d \mathcal{N}=2$ Maldacena-Nuñez solutions [18].

Holographic central charge, flavor central charge, and probe M2-branes.-The holographic central charge is extracted from the warped volume of the internal space [19],

$$
c=\frac{1}{2^{7} \pi^{6} m^{9} \ell_{p}^{9}} \int_{M_{6}} e^{9 \lambda} \operatorname{vol}_{M_{6}}=\frac{\ell N^{2} K^{2}}{12(N+K \ell)},
$$

where $\operatorname{vol}_{M_{6}}$ is the volume form of $d s_{M_{6}}^{2}$ in (1).

Expanding the $M$-theory three-form $C_{3}$ onto the resolution cycles of the $\mathbb{R}^{4} / \mathbb{Z}_{\ell}$ orbifold singularity at $\mathrm{P}_{3}$, one obtains $\ell-1$ Abelian gauge fields. The gauge group enhances to $\mathrm{SU}(\ell)$ by virtue of states from $M 2$-branes wrapping the resolution cycles [17]. We compute the associated flavor central charge $k_{\mathrm{SU}(\ell)}$ using the 't Hooft anomaly inflow methods of [20], yielding

$$
k_{\mathrm{SU}(\ell)}=\frac{2 N K \ell}{N+K \ell} .
$$

M2-brane probes wrapping calibrated two cycles in the internal space are dual to Bogomol'nyi-PrasadSommerfield (BPS) operators in the SCFT. The calibration condition was written in [19] for a generic solution preserving $4 d \mathcal{N}=1$ superconformal symmetry and can be adapted to the $\mathcal{N}=2$ solutions at hand. The conformal dimension $\Delta$ of the operator dual to an $M 2$-brane wrapping the calibrated two-cycle $\mathcal{C}_{2}$ is [19]

$$
\Delta=\frac{1}{4 \pi^{2} m^{3} \ell_{p}^{3}} \int_{\mathcal{C}_{2}} e^{3 \lambda} \operatorname{vol}_{\mathcal{C}_{2}}
$$

where $\operatorname{vol}_{\mathcal{C}_{2}}$ is the volume form on $\mathcal{C}_{2}$ induced from $d s_{M_{6}}^{2}$.

We identify two supersymmetric $M 2$-brane probes in our setup. First, we can wrap an $M 2$-brane on the $S^{2}$ on top of the orbifold point $\mathrm{P}_{3}$ in the $(w, \mu)$ plane. We denote the corresponding operator as $\mathcal{O}_{1}$. Second, we can wrap an $M 2$-brane on the $2 d$ subspace consisting of the segment $\mathrm{P}_{3} \mathrm{P}_{4}$ and the combination of $S_{\phi}^{1}$ and $S_{z}^{1}$ that does not shrink in the interior of $\mathrm{P}_{3} \mathrm{P}_{4}$. This subspace corresponds to an open M2-brane ending on the M5-branes at $w=0$. We denote the associated operator as $\mathcal{O}_{2}$. The dimensions of $\mathcal{O}_{1}, \mathcal{O}_{2}$ from (16) are

$$
\Delta\left(\mathcal{O}_{1}\right)=\frac{N K \ell}{N+K \ell}, \quad \Delta\left(\mathcal{O}_{2}\right)=K .
$$

The $\mathrm{U}(1)_{r} \times \mathrm{SU}(2)_{R}$ charges of $\mathcal{O}_{1}, \mathcal{O}_{2}$ can be computed from the $M 2$-brane coupling to the $11 d$ three-form $C_{3}$ [19],

$$
(r, R)\left(\mathcal{O}_{1}\right)=\left[2 \Delta\left(\mathcal{O}_{1}\right), 0\right], \quad(r, R)\left(\mathcal{O}_{2}\right)=\left[0, \Delta\left(\mathcal{O}_{2}\right)\right],
$$

with $R$ the Cartan generator of $\mathrm{SU}(2)_{R}$. Thus $\mathcal{O}_{1}$ and $\mathcal{O}_{2}$ have the $R$ charges of $\mathcal{N}=2$ Coulomb branch and Higgs branch operators, respectively.

A novel Stückelberg mechanism.-The Killing vector $\partial_{\beta}$ in (12) is a symmetry of the $11 d$ metric and flux, but it does not correspond to a continuous flavor symmetry of the dual SCFT. This is due to a Stuickelberg mechanism in the $5 d$ low-energy effective action of $M$ theory on $M_{6}$. The components of the $11 d$ metric with one external leg and one leg along $\partial_{\beta}$ yield a $\mathrm{U}(1)$ gauge field $A^{\beta}$. When $A^{\beta}$ is turned on, the one-form $d \beta$ must be replaced by the gauge invariant combination $d \beta+A^{\beta}$. This replacement affects the closure of $G_{4}$, which is restored by adding suitable terms, including

$$
G_{4}^{\mathrm{tot}}=\left.G_{4}\right|_{d \beta \rightarrow d \beta+A^{\beta}}+D a_{0} \wedge \omega_{3}+\cdots .
$$

The improved $G_{4}^{\text {tot }}$ is built with the closed but not exact three-form $\omega_{3} \propto l_{\partial_{\beta}} G_{4}$, whose nonexactness hinges on the $M 5$-brane source at $w=0$. The one-form $D a_{0}$ is the field strength of an external axion $a_{0}$.

Closure of $G_{4}$ requires $d D a_{0} \propto d A^{\beta}$, signaling a nontrivial Stückelberg coupling between $A^{\beta}$ and $a_{0}$. As a result, $A^{\beta}$ is massive and is dual to a spontaneously broken $\mathrm{U}(1)$ symmetry in the SCFT. As discussed in detail in [21], this mechanism provides a nontrivial physical realization of a mathematical obstruction to promoting $G_{4}$ to an equivariant cohomology class [22]. In contrast, $\iota_{\partial_{\chi}} G_{4}$ is exact, and the $\mathrm{U}(1)$ gauge field $A^{\chi}$ (originating from the components of the $11 d$ metric with one external leg and one leg along $\partial_{\chi}$ ) 
TABLE I. The central charges of the $\left(A_{N-1}^{(N)}[k], Y_{\ell}\right)$ theories. $\{x\}=x-\lfloor x\rfloor$ denotes the fractional part.

\begin{tabular}{lc}
\hline \hline$a$ & $\left\{\left[4 k^{2}\left(N^{2}-1\right)-5(k+N)\{[(8-3 \ell) / 5] N-2+\operatorname{GCD}(k, N)\}\right] /[48(k+N)]\right\}$ \\
& $+\{N /[8(k+N)]\} \sum_{j=1}^{N-1}\{[j(k+N) / N]\}(1-\{[j(k+N)] / N\})$ \\
& $+\left[\left(4 N^{3}[1-(1 / \ell)]\{2 k+N[1-(1 / \ell)]\}\right) /[48(k+N)]\right]$ \\
$c$ & $\left\{\left(k^{2}\left(N^{2}-1\right)-(k+N)[N(2-\ell)-2+\operatorname{GCD}(k, N)]\right) /[12(k+N)]\right\}$ \\
& $+\left(\left[N^{3}[1-(1 / \ell)]\{2 k+N[1-(1 / \ell)]\}\right] /[12(k+N)]\right)$ \\
$N, k \rightarrow \infty$ & $a=c=\left\{\left[N^{2}\{k+N[1-(1 / \ell)]\}^{2}\right] /[12(k+N)]\right\}$ \\
\hline \hline
\end{tabular}

does not participate in any Stückelberg coupling to $a_{0}$ and remains massless. This is expected since $\partial_{\chi}$ is dual to the $\mathrm{U}(1)_{r} R$ symmetry of the SCFT. Similar versions of the Stückelberg mechanism for isometries in flux backgrounds are known for flat internal spaces (see, e.g., [23]). The internal geometry discussed in this Letter is richer, and this Stückelberg mechanism is novel in the context of holographic $M$-theory solutions.

Field theory duals. - We claim that the $11 d$ supergravity solutions presented above are holographically dual to $4 d$ $\mathcal{N}=2$ SCFTs that arise from the low-energy limit of $N$ $M 5$-branes wrapping a sphere with an irregular puncture of type $A_{N-1}^{(N)}[k]$, labeled by the integer $k>-N$. For $\ell=1$ the irregular puncture is the only puncture on the sphere, and the $4 d$ field theories coincide with the type I theories with $b=N$ and $J=A_{N-1}$ in the classification of [24,25] (also called $I_{N, k}$ in [26]). These are the $\mathrm{AD}$ theories of type $\left(A_{N-1}, A_{k-1}\right)$, obtained in type IIB in [27] (generalizing the $N=2$ cases obtained in $[1,28,29])$. For $\ell>1$ there is an additional regular puncture at the opposite pole of the sphere that is labeled by a box Young diagram with $\ell$ columns and $N / \ell$ rows, contributing an $\mathrm{SU}(\ell)$ nonAbelian flavor symmetry [30]. We label the resulting $4 d$ theories by $\left(A_{N-1}^{(N)}[k], Y_{\ell}\right)$, which belong to the class labeled type IV in [24,25]. For $\ell=N$ the regular puncture is of maximal type and these are the $D_{k+N}^{N}[\mathrm{SU}(N)]$ theories studied in [32-34]. The case $\ell=1$ is the "nonpuncture," equivalent to the $\left(A_{N-1}, A_{k-1}\right)$ theories.

The irregular puncture is identified with the M5-brane source in the gravity dual. Because of the irregular puncture, the $\mathrm{U}(1)_{r} R$ symmetry of the SCFT is given as the combination $r=R_{\phi}+[N /(k+N)] R_{z}$, where $R_{\phi}$ is the generator of the $R$ symmetry that would be preserved in the absence of the irregular defect and $R_{z}$ is the generator of the global $\mathrm{U}(1)_{z}$ isometry of the sphere [24,25]. Comparison with (12) gives the map between $k$ in the SCFT and the flux quantum $K$,

$$
K=k+N\left(1-\frac{1}{\ell}\right)
$$

The central charges of the $\left(A_{N-1}^{(N)}[k], Y_{\ell}\right)$ theories are summarized in Table I. They are computed in the literature [24,33-36] using useful formulas from [37]. For $\ell>1$, an especially simple way to compute the central charges as a function of $\ell$ is to apply the results of [34,38] for the partial closure of a maximal puncture, initiated by a nilpotent vacuum expectation value for the moment map operator of the maximal puncture's flavor symmetry. The third row of Table I gives the central charge in the limit $N, k \rightarrow \infty$ with $k / N$ finite. Using (20), we get a perfect match with the holographic central charge (14).

The dimensions of the Coulomb branch operators $u_{i}$ of the theory $\left(A_{N-1}^{(N)}[k], Y_{\ell}\right)$ are conveniently captured by a Newton polygon [24] and obey the bounds

$$
1<\Delta\left(u_{i}\right) \leq N-\frac{N^{2}}{\ell(N+k)} .
$$

The upper bound is saturated by exactly one $u_{i}$, which has the correct dimension and $R$ charges to be identified with the $M 2$-brane operator $\mathcal{O}_{1}$ in (17), (18) [39].

Using (20), the $k_{\mathrm{SU}(\ell)}$ central charge (15) reads

$$
k_{\mathrm{SU}(\ell)}=2 N-\frac{2 N^{2}}{\ell(N+k)} .
$$

For $\ell=N$ it matches the field theory computation of [33]. For generic $\ell$, it matches the conjecture of [26] that the flavor central charge is equal to twice the maximal Coulomb branch operator dimension-see (21).

For $\ell=1$, the rank of the global symmetry of the $\left(A_{N-1}, A_{k-1}\right)$ theories is $\operatorname{GCD}(k, N)-1$, where $\mathrm{GCD}$ stands for greatest common divisor [34]. The maximal rank $N-1$ on the SCFT side matches with the maximal rank that can be achieved via the M5-brane source on the gravity side. It would be interesting to establish a precise match with the SCFT formula for generic $k, N$.

When $\ell=1$ and $k / N$ is an integer, a Lagrangian description of the SCFT was obtained in $[2,3]$ (see also $[40,41]$ for the case $N=2$ ). Using the dual Lagrangian, a set of $2^{N}-2$ Higgs branch operators can be constructed, with dimension [3]

$$
\Delta=k-\frac{k}{N} .
$$

At large $N$, this exactly matches with the dimension of the wrapped M2-brane operators $\mathcal{O}_{2}$ in (17), (18). We expect that the field-theory degeneracy factor $2^{N}-2$ could be understood on the gravity side by studying the possible 
ways in which the M2-brane can end on the M5-brane source. Heuristically, we can picture the M2-brane world volume, which has a disk topology, as the collapsed version of a multipronged configuration that can have a boundary component on each of the $N$ M5-branes independently. Since the M2-brane must end on at least one of them, the degeneracy is $2^{N}-1$. Notice the mismatch by one between the degeneracy in field theory and in gravity. It would be interesting to sharpen this argument and to understand the origin of the additional decoupled mode, which we expect is associated to the center-of-mass mode of the M5-brane source stack.

Discussion.-We have proposed gravity duals for the $4 d$ $\mathcal{N}=2$ SCFTs $\left(A_{N-1}^{(N)}[k], Y_{\ell}\right)$ of $\mathrm{AD}$ type, performing checks on the central charge, the $\mathrm{SU}(\ell)$ flavor central charge, and the dimensions of suitable Coulomb branch and Higgs branch operators. Our $\mathrm{AdS}_{5}$ solutions contain internal $M 5$-brane sources. They admit an isometry algebra $\mathfrak{S} \mathbb{a}(2)_{R} \oplus \mathfrak{t}(1)_{r} \oplus \mathfrak{t}(1)_{\beta}$. The $\mathfrak{S} \mathbb{0}(2)_{R} \oplus \mathfrak{t}(1)_{r}$ is dual to the SCFT $R$-symmetry, while $u(1)_{\beta}$ does not yield a continuous flavor symmetry thanks to a Stückelberg mechanism in which the $u(1)_{\beta}$ vector eats an axion originating from the expansion of the $M$-theory three form. There could be still a discrete symmetry remnant of $\mathfrak{u}(1)_{\beta}$, which we plan to study elsewhere.

We expect our $11 d$ solutions to admit generalizations corresponding to a regular puncture labeled by an arbitrary Young diagram. Constructing Lagrangian descriptions for these cases would yield further insights into SCFTs of AD type and allow for precision tests of the holographic duality.

It would be interesting to investigate whether the classification of irregular punctures in field theory can be recovered by a systematic study of exact solutions to the Toda equation of the class we discovered.

Our results set the stage for a broader study of holographic duals of $\mathrm{AD}$ theories. The supergravity constructions can be generalized to obtain $\mathcal{N}=1$ systems. More interestingly, our solutions can be used to study the holographic dual of the supersymmetry enhancing flows observed in the Lagrangian realizations of $\mathrm{AD}$ theories.

We are grateful to Nikolay Bobev, Simone Giacomelli, and Yifan Wang for interesting conversations and correspondence. The work of I. B. is supported in part by NSF Grant No. PHY-1820784. F. B. is supported by STFC Consolidated Grant No. ST/T000864/1. R. M. is supported in part by ERC Grant No. 787320-QBH Structure and by ERC Grant No. 772408-Stringlandscape. The work of E. N. is supported by DOE Grant No. DE-SC0020421.

[1] P. C. Argyres and M. R. Douglas, Nucl. Phys. B448, 93 (1995).

[2] P. Agarwal, A. Sciarappa, and J. Song, J. High Energy Phys. 10 (2017) 211.
[3] S. Benvenuti and S. Giacomelli, J. High Energy Phys. 10 (2017) 106.

[4] A. Brandhuber and Y. Oz, Phys. Lett. B 460, 307 (1999).

[5] F. Apruzzi, M. Fazzi, D. Rosa, and A. Tomasiello, J. High Energy Phys. 04 (2014) 064.

[6] D. Gaiotto and A. Tomasiello, J. High Energy Phys. 12 (2014) 003.

[7] F. Apruzzi, M. Fazzi, A. Passias, A. Rota, and A. Tomasiello, Phys. Rev. Lett. 115, 061601 (2015).

[8] E. D'Hoker, M. Gutperle, A. Karch, and C. F. Uhlemann, J. High Energy Phys. 08 (2016) 046.

[9] E. D’Hoker, M. Gutperle, and C. F. Uhlemann, J. High Energy Phys. 05 (2017) 131.

[10] I. Bah, A. Passias, and A. Tomasiello, J. High Energy Phys. 11 (2017) 050.

[11] I. Bah, A. Passias, and P. Weck, J. High Energy Phys. 01 (2019) 058.

[12] I. Bah, F. Bonetti, R. Minasian, and E. Nardoni (to be published).

[13] I. Bah, M. Gabella, and N. Halmagyi, J. High Energy Phys. 07 (2014) 131.

[14] P. Ferrero, J. P. Gauntlett, J. M. Pérez Ipiña, D. Martelli, and J. Sparks, Phys. Rev. Lett. 126, 111601 (2021).

[15] P. Ferrero, J. P. Gauntlett, J. M. P. Ipiña, D. Martelli, and J. Sparks, Phys. Rev. D 104, 046007 (2021).

[16] H. Lin, O. Lunin, and J. M. Maldacena, J. High Energy Phys. 10 (2004) 025.

[17] D. Gaiotto and J. Maldacena, J. High Energy Phys. 10 (2012) 189.

[18] J. M. Maldacena and C. Nunez, Int. J. Mod. Phys. A 16, 822 (2001).

[19] J. P. Gauntlett, E. O Colgain, and O. Varela, J. High Energy Phys. 02 (2007) 049.

[20] I. Bah, F. Bonetti, R. Minasian, and E. Nardoni, J. High Energy Phys. 01 (2020) 125.

[21] I. Bah, F. Bonetti, R. Minasian, and E. Nardoni, arXiv: 2106.01322.

[22] S. Wu, J. Geom. Phys. 10, 381 (1993).

[23] K. Hinterbichler, J. Levin, and C. Zukowski, Phys. Rev. D 89, 086007 (2014).

[24] D. Xie, J. High Energy Phys. 01 (2013) 100.

[25] Y. Wang and D. Xie, Phys. Rev. D 94, 065012 (2016).

[26] D. Xie and P. Zhao, J. High Energy Phys. 03 (2013) 006.

[27] S. Cecotti, A. Neitzke, and C. Vafa, arXiv:1006.3435.

[28] P. C. Argyres, M. Plesser, N. Seiberg, and E. Witten, Nucl. Phys. B461, 71 (1996).

[29] T. Eguchi, K. Hori, K. Ito, and S.-K. Yang, Nucl. Phys. B471, 430 (1996).

[30] Box Young diagrams from orbifold singularities were studied in [31].

[31] N. Bobev, P. Bomans, and F. F. Gautason, J. High Energy Phys. 06 (2020) 011.

[32] S. Cecotti and M. Del Zotto, J. High Energy Phys. 01 (2013) 191.

[33] S. Cecotti, M. Del Zotto, and S. Giacomelli, J. High Energy Phys. 04 (2013) 153.

[34] S. Giacomelli, J. High Energy Phys. 06 (2018) 156.

[35] D. Xie and S.-T. Yau, arXiv:1510.01324. 
[36] D. Xie, W. Yan, and S.-T. Yau, Phys. Rev. D 103, 065003 (2021).

[37] A. D. Shapere and Y. Tachikawa, J. High Energy Phys. 09 (2008) 109.

[38] S. Giacomelli, N. Mekareeya, and M. Sacchi, J. High Energy Phys. 03 (2021) 242.
[39] A Coulomb branch operator has $\mathrm{U}(1)_{r}$ charge $r=2 \Delta$ and is uncharged under $\mathrm{SU}(2)_{R}$.

[40] K. Maruyoshi and J. Song, Phys. Rev. Lett. 118, 151602 (2017).

[41] K. Maruyoshi and J. Song, J. High Energy Phys. 02 (2017) 075. 